\begin{tabular}{|c|c|}
\hline Title & Theoretical and experimental results of electronic transport of spin quantum cross structure devices \\
\hline Author(s) & Kondo, Kenji; Kaiju, Hideo; Ishibashi, A kira \\
\hline Citation & $\begin{array}{l}\text { Journal of applied physics, 105(7), 07D522-1-07D522-3 } \\
\text { https://doi.org/10.1063/1.3072781 }\end{array}$ \\
\hline Issue Date & 2009-04-01 \\
\hline Doc URL & http:/hdl.handle.net/2115/56989 \\
\hline Rights & $\begin{array}{l}\text { Copyright } 2009 \text { A merican Institute of Physics. This article may be downloaded for personal use only. A ny other use } \\
\text { requires prior permission of the author and the A merican Institute of Physics. The following article appeared in Journal } \\
\text { of A pplied Physics and may be found at http://scitation.aip.org/content/aip } \$ \text { ournal } / 2 a p / 105 / 1 / 10.1063 / 1.3072781 \text {. }\end{array}$ \\
\hline Type & article \\
\hline File Information & JAP_105_07D522_1.3072781.pdf \\
\hline
\end{tabular}

Instructions for use 


\title{
Theoretical and experimental results of electronic transport of spin quantum cross structure devices
}

\author{
Kenji Kondo, ${ }^{\text {a) }}$ Hideo Kaiju, and Akira Ishibashi \\ Laboratory of Quantum Electronics, Research Institute for Electronic Science, Hokkaido University, \\ Sapporo, Hokkaido 001-0020, Japan
}

(Presented 12 November 2008; received 17 September 2008; accepted 25 November 2008; published online 25 February 2009)

\begin{abstract}
Recently, we have proposed quantum cross structure (QCS) devices that consist of two metal thin films deposited on organic films with edge-to-edge configuration like crossed fins for switching devices. In this paper, we propose a spin quantum cross structure (SQCS) device, which is a QCS device consisting of two magnetic thin films. We show theoretical and experimental results of electronic transport characteristics regarding SQCS devices. The calculation of the $I-V$ characteristics has been performed for the SQCS devices with the Ni magnetic thin films for both the electrodes within the framework of the Anderson model. Then, we fabricated a SQCS device with the $\mathrm{Ni}$ magnetic thin films and measured the $I-V$ characteristics by a four-terminal method. Also, the calculation of the magnetoresistance ratio has been done as a function of renormalized transfer matrices including magnetostriction effects and the other effects phenomenologically.
\end{abstract}

(C) 2009 American Institute of Physics. [DOI: 10.1063/1.3072781]

Recently, we have proposed a double "nanobaumkuchen" (DNB) structure, composed of two thin slices of alternating metal/insulator nanobaumkuchen, as a lithography-free nanostructure fabrication technology ${ }^{1-3}$ and a quantum cross structure (QCS) device. ${ }^{3,4}$ The cross point of the DNB can be scaled down to ultimate feature sizes of a few nanometers due to the film thickness determined by the metal-deposition rate, ranging from 0.01 to $1 \mathrm{~nm} / \mathrm{s}$. We call one crossing element of the DNB structure a QCS device that consists of two metal nanoribbons with the edge-to-edge configuration. Especially, we call the QCS device with magnetic electrodes or spin injection mechanism "a spin quantum cross structure (SQCS) device," shown in Fig. 1(a).

We study the current-voltage characteristics of SQCS devices with a molecule sandwiched between two Ni magnetic electrodes. The molecule is assumed to have two energy levels. We also assume that the molecule does not flip the spin of an electron passing through the energy level. The model of SQCS devices with two-dimensional (2D) electrodes is shown in Fig. 1(b). The energy diagram for the SQCS device model is also shown in Fig. 1(c). We analyze the transport characteristics using the Anderson Hamiltonian. The Anderson Hamiltonian is described as follows:

$$
H=H_{\text {Electrodes }}+H_{\text {mole }}+H_{t},
$$

where

$$
\begin{aligned}
& H_{\text {Electrodes }}=\sum_{\alpha=L, R} \sum_{\boldsymbol{k}, \sigma} \epsilon_{k \sigma} c_{\alpha, \boldsymbol{k} \sigma}^{\dagger} c_{\alpha, \boldsymbol{k} \sigma}, \\
& H_{\text {mole }}=\sum_{i, \sigma} \epsilon_{0}(i) a_{i, \sigma}^{\dagger} a_{i, \sigma},
\end{aligned}
$$

${ }^{a)}$ Electronic mail: kkondo@es.hokudai.ac.jp.

$$
H_{t}=\sum_{\alpha=L, R} \sum_{k, \sigma} \sum_{i, \sigma}\left(V_{\alpha}^{\sigma} c_{\alpha, \boldsymbol{k} \sigma}^{\dagger} a_{i, \sigma}+\text { H.c. }\right) .
$$

$H_{\text {Electrodes }}$ is the Hamiltonian of both the Ni electrodes, $\epsilon_{k \sigma}$ $=\hbar^{2} \boldsymbol{k}^{2} / 2 m, m$ is the free electron mass, and $\hbar$ is the Planck constant divided by $2 \pi$. The band of itinerant $d$-like electrons of $\mathrm{Ni}$ is assumed to be parabolic with the free electron mass. ${ }^{5}$ The wave vector $\boldsymbol{k}$ is a $2 \mathrm{D}$ vector in $2 \mathrm{D}$ electrodes. $c_{\alpha, k \sigma}^{\dagger}$ and $c_{\alpha, k \sigma}$ are creation and annihilation operators for electrons of wave vector $\boldsymbol{k}$ and spin index $\sigma$ in $\alpha$ electrode. $\alpha$ indicates the left or right electrode. The creation and annihilation operators obey the standard fermion anticommunication rules. $H_{\text {mole }}$ is the Hamiltonian of a molecule sandwiched between both electrodes, and $\epsilon_{0}(i)$ represents the $i$ th energy level of eigenstates of the molecule as shown in Fig. 1(c). The two energy levels are $\epsilon_{0}(1)=0.5 \mathrm{eV}$ and $\epsilon_{0}(2)$ $=1 \mathrm{eV}$, estimated from Fermi levels $E_{F L}$ and $E_{F R}$ of the two electrodes, respectively. The Fermi levels $E_{F L}$ and $E_{F R}$ of the electrodes were assumed to be equal, and we used the value of $9.071 \mathrm{eV}$ of $\mathrm{Ni}$ for the Fermi level. ${ }^{6}$ The value of 260 $\mathrm{meV}$ was used for the exchange splitting of $\mathrm{Ni}^{7}{ }^{7} a_{i, \sigma}^{\dagger}$ and $a_{i, \sigma}$ are creation and annihilation operators for electrons of spin index $\sigma$ in the $i$ th energy level. These operators also obey the standard fermion anticommunication rules. $H_{t}$ is the transfer Hamiltonian between the sandwiched molecule and each electrode. $V_{\alpha}^{\sigma}$ is the spin-dependent transfer matrix between the molecule and electrons of spin index $\sigma$ in $\alpha$ electrode. This value determines the coupling strength between each electrode and the molecule. Considering $H_{t}$ as a perturbation, we have investigated the current-voltage characteristics from right to left electrode by changing these coupling strengths. The electrons flow from left to right electrode. After tedious manipulation, the current $I$ from right to left electrode is as follows: 


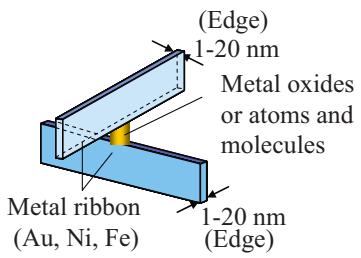

(a) Spin quantum cross structure devices

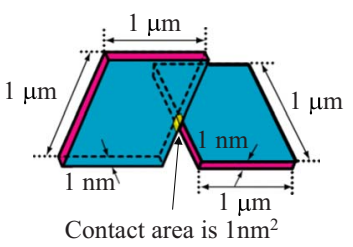

(b) SQCS model with 2D electrodes

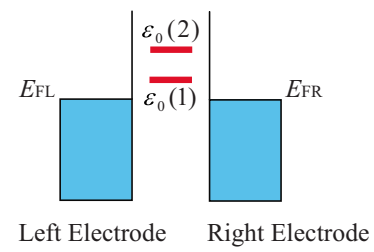

(c) Energy diagram for SQCS devices
FIG. 1. (Color online) Schematic illustrations of (a) SQCS devices and (b) SQCS device model with $2 \mathrm{D} \mathrm{Ni}$ electrodes. (c) The energy diagram for SQCS devices.

$$
I=e\left(P_{L \rightarrow R}-P_{R \rightarrow L}\right)= \begin{cases}I_{\uparrow \uparrow}, & \text { parallel } \\ I_{\uparrow \downarrow}, & \text { antiparallel, }\end{cases}
$$

where $e$ is the elementary charge, $P_{L \rightarrow R}\left(P_{R \rightarrow L}\right)$ is the transition probability of electrons from left to right (right to left) electrode, and $I_{\uparrow \uparrow}\left(I_{\uparrow \downarrow}\right)$ is the current flowing from right to left electrode when two Ni magnetic electrodes have the parallel (antiparallel) magnetic moments. They are given by

$$
\begin{aligned}
I_{\uparrow \uparrow}= & \frac{e^{2}}{h} \int_{E_{F R}}^{E_{F R}+e V} d \epsilon \sum_{i}\left(\frac{4 \Gamma_{L}^{+}(\epsilon) \Gamma_{R}^{+}(\epsilon)}{\left[\epsilon-\epsilon_{0}(i)\right]^{2}+\left[\Gamma^{++}(\epsilon)\right]^{2}}\right. \\
& \left.+\frac{4 \Gamma_{L}^{-}(\epsilon) \Gamma_{R}^{-}(\epsilon)}{\left[\epsilon-\epsilon_{0}(i)\right]^{2}+\left[\Gamma^{--}(\epsilon)\right]^{2}}\right) \\
& \times\left[f\left(\epsilon-e V-E_{F R}\right)-f\left(\epsilon-E_{F R}\right)\right], \\
I_{\uparrow \downarrow}= & \frac{e^{2}}{h} \int_{E_{F R}}^{E_{F R}+e V} d \epsilon \sum_{i}\left(\frac{4 \Gamma_{L}^{+}(\epsilon) \Gamma_{R}^{-}(\epsilon)}{\left[\epsilon-\epsilon_{0}(i)\right]^{2}+\left[\Gamma^{+-}(\epsilon)\right]^{2}}\right. \\
& \left.+\frac{4 \Gamma_{L}^{-}(\epsilon) \Gamma_{R}^{+}(\epsilon)}{\left[\epsilon-\epsilon_{0}(i)\right]^{2}+\left[\Gamma^{-+}(\epsilon)\right]^{2}}\right) \\
& \times\left[f\left(\epsilon-e V-E_{F R}\right)-f\left(\epsilon-E_{F R}\right)\right],
\end{aligned}
$$

where $f(\epsilon)$ is the Fermi-Dirac distribution function, $\Gamma^{++}(\epsilon)$ $=\Gamma_{L}^{+}(\epsilon)+\Gamma_{R}^{+}(\epsilon), \Gamma^{--}(\epsilon)=\Gamma_{L}^{-}(\epsilon)+\Gamma_{R}^{-}(\epsilon), \Gamma^{+-}(\epsilon)=\Gamma_{L}^{+}(\epsilon)+\Gamma_{R}^{-}(\epsilon)$, and $\Gamma^{-+}(\epsilon)=\Gamma_{L}^{-}(\epsilon)+\Gamma_{R}^{+}(\epsilon)$. Since both the electrodes are the same $\mathrm{Ni}$, the following equations hold from the symmetry:

$$
\Gamma^{+-}(\epsilon)=\Gamma^{-+}(\epsilon), \quad \Gamma_{L}^{+}(\epsilon)=\Gamma_{R}^{+}(\epsilon), \quad \Gamma_{L}^{-}(\epsilon)=\Gamma_{R}^{-}(\epsilon)
$$

The superscript $+(-)$ designates the coupling between the density of states of the majority (minority) spin electrons and the molecule. Then, $\Gamma_{L}^{+}(\epsilon)$ and $\Gamma_{R}^{+}(\epsilon)\left[\Gamma_{L}^{-}(\epsilon)\right.$ and $\left.\Gamma_{R}^{-}(\epsilon)\right]$ are coupling strengths between the molecule and the majority (minority) spin electrons of left and right electrodes, respectively, which are given in the following:

$$
\begin{array}{ll}
\Gamma_{L}^{+}(\epsilon)=\pi D_{L}^{+}(\epsilon)\left|V_{L}^{+}\right|^{2}, & \Gamma_{L}^{-}(\epsilon)=\pi D_{L}^{-}(\epsilon)\left|V_{L}^{-}\right|^{2} \\
\Gamma_{R}^{+}(\epsilon)=\pi D_{R}^{+}(\epsilon)\left|V_{R}^{+}\right|^{2}, & \Gamma_{R}^{-}(\epsilon)=\pi D_{R}^{-}(\epsilon)\left|V_{R}^{-}\right|^{2},
\end{array}
$$

where $D_{L}^{+}(\epsilon)$ and $D_{R}^{+}(\epsilon)\left[D_{L}^{-}(\epsilon)\right.$ and $\left.D_{R}^{-}(\epsilon)\right]$ are densities of states of the majority (minority) spin electrons in left and right electrodes, respectively, and $V_{L}^{+}$and $V_{R}^{+}\left(V_{L}^{-}\right.$and $\left.V_{R}^{-}\right)$are transfer matrices between the molecule and majority (minority) spin electrons in left and right electrodes, respectively. In this model, the relations $V_{L}^{+}=V_{R}^{+}$and $V_{L}=V_{R}^{-}$hold from the symmetry. Now, we define the renormalized transfer matrices as follows:

$$
W_{L(R)}^{+(-)}(\epsilon)=D_{L(R)}^{+(-)}(\epsilon)\left|V_{L(R)}^{+(-)}\right|^{2} .
$$

These renormalized transfer matrices can include the spin polarization effects, physical disconnection induced by magnetostriction effects, and the other effects with application of magnetic field. In our model, the MR ratio is given by

$$
\mathrm{MR}=\frac{G_{\uparrow \uparrow}-G_{\uparrow \downarrow}}{G_{\uparrow \uparrow}}=\left.\frac{I_{\uparrow \uparrow}-I_{\uparrow \downarrow}}{I_{\uparrow \uparrow}}\right|_{V=V_{0}},
$$

where $G_{\uparrow \uparrow}\left(G_{\uparrow \downarrow}\right)$ is a conductance of SQCS devices when two $\mathrm{Ni}$ magnetic electrodes have the parallel (antiparallel) magnetic moments. They are dependent on the applied voltage. Then, MR ratio is defined at $V_{0} . V_{0}$ is the operating voltage, which is $0.75 \mathrm{~V}$ in this calculation. We have calculated the transport characteristics at room temperature of 300 $\mathrm{K}$. Figure 2(a) shows the $I-V$ characteristics of SQCS devices with two Ni thin film electrodes with parallel magnetic moments under the weak coupling condition. We regard the coupling as the weak coupling when the energy of coupling strength is smaller than that of the ambient temperature 27 meV. In this situation, we used the coupling strengths $\Gamma_{L}^{+}(\epsilon)$ and $\Gamma_{R}^{+}(\epsilon)$ of $1.57 \mathrm{meV}$. The results show sharp steps at the positions of the energy level of the sandwiched molecule. Even at room temperature, these conductance peaks are sharp, indicating that a SQCS device with Ni thin film electrodes can be used as a switching device that works at very small voltage change. Figure 2(b) shows the $I-V$ characteristics of SQCS devices under the strong coupling limit. The strong coupling limit means that the energies of the coupling strengths $\Gamma_{L}^{+}(\epsilon)$ and $\Gamma_{R}^{+}(\epsilon)$ are much larger than that of the ambient temperature. We used the coupling strengths $\Gamma_{L}^{+}(\epsilon)$ and $\Gamma_{R}^{+}(\epsilon)$ of $3927 \mathrm{meV}$. In this case, the energy level of the sandwiched molecule is almost continuous because of the wide broadening induced by the strong coupling with the itinerant electrons. Therefore, we expect the $I-V$ characteristics to be like those of the Ohmic contact without the molecule. As expected, we can obtain the Ohmic-type $I-V$ characteristics. The MR ratio calculated by Eq. (9) is shown in Fig. 3(a). We have calculated the MR ratio with the renor-
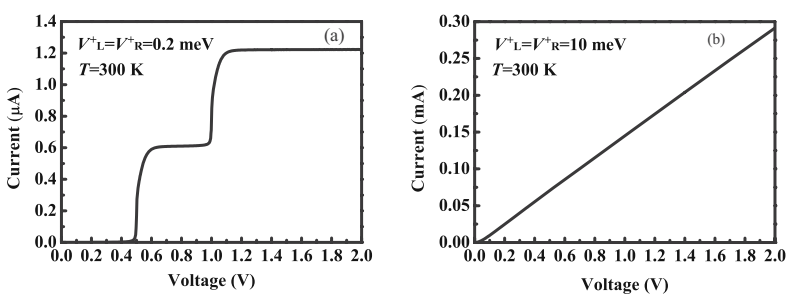

FIG. 2. The current-voltage characteristics of SQCS devices (a) under the weak coupling condition and (b) under the strong coupling limit. 

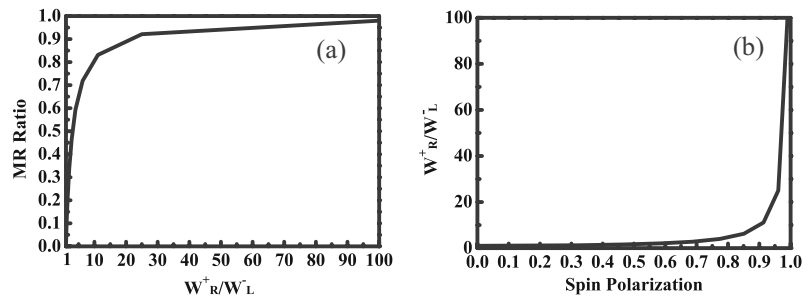

FIG. 3. (a) The calculated MR ratio of SQCS devices. (b) The relation between the renormalized transfer matrices and the spin polarizations of ferromagnetic electrodes of SQCS devices.

malized transfer matrices changed while imagining that SQCS devices have ferromagnetic materials with different spin polarizations in both the electrodes, and that the coupling between both the electrodes is getting weak due to physical shrinkage induced by magnetostriction effects with application of magnetic field. The calculated curve is described very well by the following fitting function:

$$
\mathrm{MR}=\frac{\left(\frac{W_{R}^{+}}{W_{L}^{-}}\right)-1}{\left(\frac{W_{R}^{+}}{W_{L}^{-}}\right)+1} .
$$

We notice that this function is very similar to the amplitude reflection coefficient of electromagnetic waves, regarding the renormalized transfer matrix ratio as a relative refractive index. Namely, the MR ratio can be regarded as an amplitude reflection coefficient of itinerant $d$-like electrons from one electrode to the other electrode. When we know the spin polarizations of the ferromagnetic electrodes, the MR ratio can be calculated by the Julliere model. ${ }^{8}$ Though our calculation results include not only spin polarization effects but also the other effects with application of magnetic field, we apply the Julliere formula to the calculation results in order to derive the relation between the renormalized transfer matrices and the spin polarization of ferromagnetic electrodes. The relation is shown in Fig. 3(b). If you know the spin polarization of magnetic electrodes, you can obtain the ratio of the renormalized transfer matrices from Fig. 3(b). Then, the expected MR ratio can be obtained from Fig. 3(a), using the ratio of the renormalized transfer matrices. If the experimental MR ratio is higher than the value, we can suggest that the other effects such as magnetostriction enhance the MR ratio.

We have realized a prototype SQCS device with Ni thin film electrodes. Ni thin films have been thermally evaporated on polyethylene naphthalate (PEN) substrates $(2 \mathrm{~mm}$ width, $10 \mathrm{~mm}$ length, and $100 \mu \mathrm{m}$ thickness) in a high vacuum chamber at the base pressure of $10^{-8}$ Torr. The growth rate is $0.93 \mathrm{~nm} / \mathrm{min}$ at the evaporation power of $280 \mathrm{~W}$. Fabri-

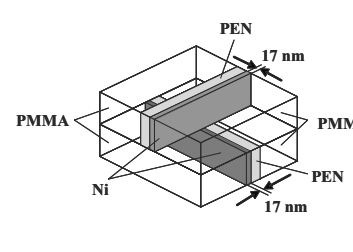

(a) A fabricated SQCS device (b) Experimental current-voltage characteristics

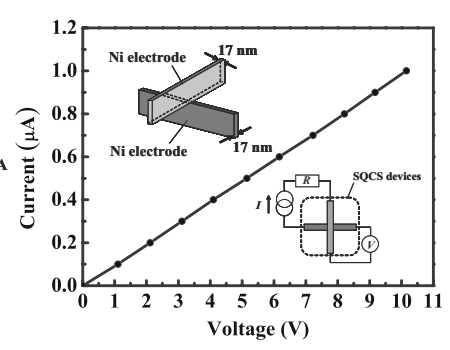

FIG. 4. (a) A fabricated SQCS device. (b) Experimental current-voltage characteristics of SQCS devices and the measurement setup of the fourprobe method.

cated Ni/PEN films have been sandwiched between two polymethyl methacrylate (PMMA) resins. The edge of PMMA/Ni/PEN/PMMA has been polished by chemical mechanical polishing methods using alumina $\left(\mathrm{Al}_{2} \mathrm{O}_{3}\right)$ slurries with particle diameters of $0.1,0.3$, and $1.0 \mu \mathrm{m}$. Two sets of polished PMMA/Ni/PEN/PMMA have been prepared and attached together with their edges crossing in a highly clean environment. ${ }^{9}$ Figure 4(a) shows the schematic illustration of a fabricated SQCS device consisting of two Ni thin films. The Ni thickness measured by an optical method is $17 \mathrm{~nm}$. Since the Ni thickness is $17 \mathrm{~nm}$, the cross-sectional area between two Ni thin films is $17 \times 17 \mathrm{~nm}^{2}$. The experimental current-voltage characteristics of the SQCS device have been measured by a four-probe method at room temperature, and are shown in Fig. 4(b). The experimental results agree well with the theoretical one in Fig. 2(b) qualitatively. The measured resistance is very large and cannot be explained within the above theory. We suspect that this discrepancy is attributed to the resistance of the electrodes themselves. We also measured the MR effect of the fabricated SQCS device, but the MR effect was not observed. We consider that this is because the measured very large resistance prevents us from observing the small MR effect.

This research was partially supported by Special Education and Research Expenses from Post-Silicon Materials and Devices Research Alliance.

${ }^{1}$ A. Ishibashi, Proceedings of International Symposium on Nano Science and Technology, Tainan, Taiwan, 20-21 November 2004, p. 44.

${ }^{2}$ H. Kaiju, A. Ono, N. Kawaguchi, and A. Ishibashi, Jpn. J. Appl. Phys. 47, 244 (2008).

${ }^{3}$ K. Kondo, H. Kaiju, and A. Ishibashi, Mater. Res. Soc. Symp. Proc. 1067, B03011 (2008).

${ }^{4}$ K. Kondo and A. Ishibashi, Jpn. J. Appl. Phys., Part 1 45, 9137 (2006).

${ }^{5}$ M. B. Stearns, J. Magn. Magn. Mater. 5, 167 (1977).

${ }^{6}$ C. S. Wang and J. Callaway, Phys. Rev. B 15, 298 (1977).

${ }^{7}$ W. Eberhardt and E. W. Plummer, Phys. Rev. B 21, 3245 (1980).

${ }^{8}$ M. Julliere, Phys. Lett. 54A, 225 (1975).

${ }^{9}$ D. Md Rahaman, K. Gomita, N. Kawaguchi, H. Kaiju, K. Kondo, and A. Ishibashi, Electron. Lett. 43, 1356 (2007). 